\title{
GAMIEFECTS: A PROPOSTA DE UM FRAMEWORK PARA APOIAR A AVALIAÇÃO DE GAMIFICAÇÃO EM CONTEXTOS EDUCACIONAIS
}

Wendel Souto Reinheimer, UFSM, wsreinheimer@inf.ufsm.br, https://orcid.org/00000001-6142-6572

Roseclea Duarte Medina, UFSM, rose@inf.ufsm.br, https://orcid.org/0000-0001-61426572

Resumo. Na educação, a gamificação tem se mostrado uma estratégia promissora para promover o aprendizado, a motivação e o engajamento dos alunos. Entretanto, alguns estudos têm mostrado que seu uso nem sempre traz resultados positivos, sendo imprescindível considerar o processo de avaliação um aspecto importante, uma vez que a partir dela é possível verificar a eficácia da gamificação e se os objetivos da gamificação estão sendo alcançados. Nesse contexto, verificou-se a escassez de métodos ou abordagens avaliativas específicas para a gamificação, resultando na falta de instruções e orientações para a realização desse procedimento. Assim, este trabalho trata-se de parte de uma dissertação em fase desenvolvimento, e apresenta um framework para apoiar o processo de avaliação da gamificação em contextos educacionais. O framework GAMIEFECTS foi desenvolvido com base no Design Science Research Methodology (DSRM) e é composto por cinco dimensões, onde cada uma delas corresponde a um determinado aspecto de avaliação e deve ser capaz de auxiliar professores, pesquisadores e/ou designers com processos avaliativos que envolvem o uso da gamificação para contextos de aprendizagem.

Palavras-chaves: Gamificação, Framework, Avaliação, Educação, GAMIEFECTS.

\section{GAMIEFECTS: THE PROPOSAL OF A FRAMEWORK TO SUPPORT GAMIFICATION ASSESSMENT IN EDUCATIONAL CONTEXTS}

Summary. In education, gamification has proven to be a promising strategy to promote student learning, motivation and engagement. However, some studies have shown that its use does not always bring positive results, and it is essential to consider the evaluation process an important aspect, since from it is possible to verify the effectiveness of gamification and whether the gamification objectives are being achieved. In this context, there was a scarcity of methods or specific evaluative approaches for gamification, resulting in a lack of instructions and guidelines for performing this procedure. Thus, this work is part of a dissertation under development, and presents a framework to support the evaluation process of gamification in educational contexts. The GAMIEFECTS framework was developed based on the Design Science Research Methodology (DSRM) and is composed of five dimensions, each of which corresponds to a certain assessment aspect and must be able to assist teachers, researchers and/or designers with assessment processes that involve the use of gamification for learning contexts.

Keywords: Gamification, Framework, Assessment, Education, GAMIEFECTS.

\section{Introdução: Uma visão geral sobre avaliação da gamificação na educação}

No contexto educacional, um padrão comum na maioria das pesquisas que envolvem o uso de gamificação em contextos de aprendizagem consiste em projetar e desenvolver um curso, atividade ou ambiente gamificado, testá-lo em um experimento e avaliar os ganhos de desempenho nos estudantes (Dichev e Dicheva, 2017). Contudo, apesar da inclinação da temática com a área educação, na literatura é possível encontrar diversas pesquisas 
empíricas que apresentam algum tipo de limitação ou resultado inconclusivo perante as expectativas de sua implementação.

As pesquisas empíricas de De-Marcos et al., (2013) e Hanus e Fox (2015) demonstram que a utilização de elementos de jogos competitivos (e.g., badges, leaderboards e points) trouxe resultados indesejáveis, desmotivando os alunos e prejudicando sua aprendizagem. Roy e Zaman (2017) argumenta que resultados insatisfatórios muitas vezes podem estar correlacionados a fatores como mal planejamento/design do ambiente gamificado ou em decorrência da ausência de teorias pedagógicas sólidas, por outro lado, o autor destaca que os resultados inconclusivos buscam justificar que os efeitos motivacionais da gamificação são causados apenas pelo efeito de novidade e, portanto, são temporários.

Corroborando com esse cenário, Toda et al., (2018) argumenta que a gamificação tem atraído a atenção de professores que, por vezes, não possuem tempo, recursos ou conhecimentos necessários sobre gamificação para planejar e executar as atividades gamificadas, isto é, são professores em atividades que possuem formação primária em outras áreas do conhecimento, mas que possuem interesse em aplicar projetos de gamificação em suas aulas visando alcançar melhores resultados para a aprendizagem ou outros aspectos motivacionais/comportamentais nos alunos. Diante disso, buscando promover um maior entendimento sobre o estado da arte da avaliação da gamificação, serão abordadas algumas revisões de literatura que se debruçaram em investigar aspectos relacionados ao processo de avaliação da gamificação em contextos educacionais.

A pesquisa de Ogawa et al., (2016), constatou que a gamificação é avaliada a partir de diferentes procedimentos, incluindo a aplicação de Exercícios ou Tarefas, Questionários, Teste e Exames, Fórum de discussão, Download de materiais e Projetos. Com base nestes procedimentos avaliativos, verificou-se que as métricas mais utilizadas estão relacionadas ao desempenho, sendo avaliado através das notas/pontuações, conclusão e acertos em tarefas e exercícios. Ainda, os autores identificaram quatro métricas referentes às características pessoais dos estudantes, sendo estas: motivação, grau de satisfação, engajamento e assiduidade.

O estudo de Dichev e Dicheva (2017) relata que a maioria das pesquisas empíricas de gamificação na educação realizam a avaliação da gamificação a partir dos resultados ou efeitos causados nos estudantes. Além disso, por se tratar de uma revisão crítica de literatura, os autores realizam diversos apontamentos e limitações acercas dos estudos investigados. Para os autores, apesar de haver um número crescente de estudos relatando evidências empíricas sobre a efetividade da gamificação na educação, constatou-se também que uma série de pesquisas apresentam evidências inconclusivas ou insuficientes para fazer afirmações válidas sobre a eficácia da gamificação. Os autores atribuem isso a duas causas principais, a primeira é o Hype para publicar sobre a gamificação, enquanto a segunda é a aplicação de questões de pesquisa muito amplas com suporte em evidências limitadas. Para os autores, questões como "se a gamificação motivou os alunos", "se a gamificação melhorou o aprendizado" ou se a "gamificação aumentou a participação" são muito amplas. Assim, é sugerido que o foco seja restrito a perguntas mais específicas, como se o uso de determinado elemento de jogo é eficaz para um determinado tipo de estudante que participa de um determinado tipo de atividade.

$\mathrm{Na}$ pesquisa de Klock et al., (2018), os autores classificaram os tipos de avaliação em quatro categorias: interação, desempenho, experiência do usuário e gamificação. Na categoria de interação, as métricas de avaliação identificadas foram: participação em fórum, presença em aula, submissão de atividades, tempo de resposta, download, entre 
outros. Já na categoria de desempenho, as métricas identificadas foram deadlines e notas. A terceira categoria, classificada como experiência do usuário, agrupou métricas relacionadas aos estudantes como satisfação, engajamento e motivação. Por fim, a categoria gamificação foi avaliada através do ganho de pontos e distintivos. Os autores constataram que para a avaliação das métricas citadas, os estudos investigados analisam entre 2 e 3 tarefas realizadas pelos estudantes em um determinado percurso pedagógico.

Corroborando com as demais pesquisas, o mapeamento sistemático de Reinheimer e Medina (2020) mostra que a gamificação tem sido avaliada a partir de diversos critérios relacionados à aprendizagem (e.g., notas, acertos em questões, pontuação em atividades), à participação (e.g., engajamento e interação nas atividades e nos ambientes gamificados) e também com base na percepção dos usuários (e.g., respostas atribuídas através da opinião dos participantes com base em questionários, entrevistas, grupos focais). Além disso, os autores destacam a baixa adesão de soluções (e.g., frameworks, instrumentos ou escalas validados especificamente desenvolvidas para avaliar gamificação), sendo uma área pouco explorada e desenvolvida. Os autores argumentam que apesar dos estudos utilizarem instrumentos validados em suas avaliações, a maioria deles não foi elaborado exclusivamente para gamificação, sendo possível perceber a ausência de métodos de avaliação específicos para avaliar

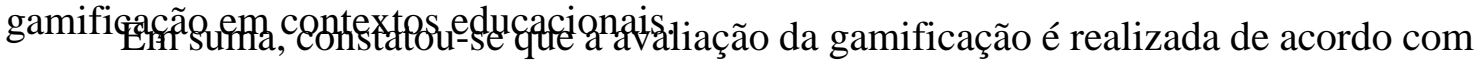
o contexto do estudo no qual a gamificação foi aplicada, ou seja, apesar de existirem semelhanças no processo de avaliação entre as pesquisas, cada pesquisador aplica a avaliação de acordo com as suas necessidades; outro aspecto que se destaca é que nem sempre os resultados relatados estão de acordo com os objetivos da pesquisa, em alguns casos, carecendo de evidências empíricas. Portanto, visando fomentar esse cenário, este trabalho tem como finalidade apresentar o desenvolvimento de um framework com a finalidade de instruir e orientar possíveis avaliadores (e.g., professores, designers e pesquisadores) no processo de avaliação da gamificação em contextos educacionais. Diante disso, este artigo está organizado em quatro seções, incluindo esta introdução. Na segunda seção, é apresentada a metodologia adotada para o desenvolvimento do framework; a terceira seção é composta pelo desenvolvimento do framework e por fim, a última seção apresenta as discussões e futuros trabalhos envolvendo o uso do framework.

\section{Metodologia de pesquisa}

Os procedimentos metodológicos previstos para a elaboração do framework baseiam-se na metodologia de pesquisa denominada Design Science Research Methodology (DSRM), desenvolvida por Peffers et al., (2007), proposta para elaboração de pesquisas científicas em sistemas de informação. Segundo o autor, ao seguir o método proposto deve ser possível compreender o estado da arte de um determinado campo de atividade, desenvolver, comunicar e avaliar os resultados da pesquisa.

É imprescindível mencionar que o desenvolvimento deste trabalho faz parte de um projeto de dissertação, portanto, algumas das etapas anteriores que correspondem ao DSRM já foram desenvolvidas, sendo estas, a primeira e segunda etapa que correspondem, respectivamente, a identificação do problema e definição dos objetivos. Para a identificação do problema, desenvolveu-se um mapeamento sistemático de literatura centrado na avaliação da gamificação em contextos de aprendizagem - que pode ser visto em Reinheimer e Medina (2020) - assim como revisões na literatura especializada de gamificação. Baseado nesse estudo, definiu-se como principal objetivo 
de pesquisa elaborar um framework para apoiar o processo de avaliação da gamificação em contextos de aprendizagem.

Portanto, a terceira etapa, denominada projeto e desenvolvimento é efetivamente a construção do artefato. Segundo o autor, essa etapa inclui determinar a funcionalidade desejada do artefato e sua arquitetura. Para tal, o artefato - Framework proposto desenvolvido nesse trabalho tem como finalidade orientar avaliadores no processo de avaliação da gamificação em contextos educacionais e pode ser classificado como um método. Segundo Costa e Silva (2014) os artefatos são classificados como métodos quando representam um conjunto de passos, algoritmo ou um guia para desempenhar uma tarefa específica. Assim, a estrutura do framework proposto foi fortemente inspirada pelo framework de Rogers et al., (2013), que apresenta uma estrutura voltada para a construção de avaliações para área da IHC (Interação Humano-Computador). Não obstante, o framework também foi elaborado com contribuições provenientes de diversos estudos na literatura especializada sobre gamificação na educação.

\section{O Framework GAMIEFECTS: uma visão geral}

Seguindo o mesmo princípio do framework DECIDE de Rogers et al., (2013), o Framework GAMIEFECTS (do acrônimo, Gamification Evaluation For Education Contexts), tem como principal finalidade orientar e guiar professores, desenvolvedores e/ou pesquisadores a realizarem a avaliação de gamificação em ambientes educacionais, considerando processos que envolvem o planejamento da avaliação, a aplicação, a coleta de dados e a apresentação dos resultados coletados. Apesar de ter sua estrutura inspirada no framework DECIDE, as informações contidas no GAMIEFECTS foram, em sua maioria, extraídas de trabalhos da área de gamificação, portanto, cada dimensão apresenta um conjunto de informações e diretrizes que atuam a fim de guiar o avaliador ao longo da avaliação. Como resultado, o GAMIEFECTS é composto por cinco dimensões distintas, mas complementares entre si.

A Dimensão 1 corresponde aos objetivos e métricas que podem ser considerados ao definir uma avaliação. A Dimensão 2 trata da análise do contexto, onde são apresentados alguns elementos que podem estar relacionados ao contex to da aplicação da gamificação e do escopo da avaliação. Na Dimensão 3 são discutidos os procedimentos a serem realizados durante a aplicação da avaliação. Na Dimensão 4 são descritas algumas questões éticas que devem ser consideradas quando a avaliação envolver outros sujeitos. Por fim, a Dimensão 5 apresenta os elementos que envolvem a análise, interpretação e apresentação dos resultados da avaliação.

\subsection{Dimensão 1 - O Que Avaliar? \\ - Diretriz 1: Definir um ou mais objetivos para a avaliação e identificar possíveis métricas de medição}

O objetivo da primeira dimensão é auxiliar o avaliador a determinar os objetivos da avaliação. No contexto educacional, a gamificação vem sendo implementada, principalmente, buscando promover a aprendizagem dos conteúdos, promovendo a interação dos usuários com o ambiente gamificado (i.e., estimular a interação do usuário através dos elementos de jogos que foram implementados) e com outros usuários. Apesar disso, os objetivos da avaliação da gamificação não precisam estar relacionados apenas com critérios de aprendizagem ou interação do usuário. Portanto, compreender e definir o que se deseja avaliar é fundamental para planejar o restante da avaliação.

Por se tratar de uma temática recente, a maioria das avaliações propostas em frameworks de gamificação ainda são centradas no usuário, como é o caso do framework 5W2H (Klock et al., 2016) que discute a avaliação da gamificação a partir dos efeitos que V. $19 \mathrm{~N}^{\circ} 2$, Dezembro, 2021 RENOTE 
a mesma causa sobre os usuários finais com base nos comportamentos desejados pelo seu uma avaliação voltada para a aprendizagem com base em testes de aceitação, isto é, testes: que tem como finalidade verificar se os objetivos a partir do uso de gamificação foram alcançados através de indicadores de desempenho (e.g., o aluno deve atingir media $7 \mathrm{de}$ um total de 10 na unidade curricular e entregar todos as tarefas propostas). Outra abordagem que se destaca na literatura de gamificação sugere a avaliação com base em critérios provenientes da área de jogos (Nah et al., 2013). Finalmente, é possível encontrar inúmeros estudos que avaliam a gamificação enquanto aplicação, ou seja, avaliando aspectos relacionadas à interface de ambientes gamificados (e.g., avaliações de usabilidade, ergonomia, entre outros) ou também verificando o funcionamento correto do sistema. Entretanto, foi possível perceber que a literatura ainda carece de avaliações especificas da área de gamificação (e.g, design da gamificação, análise dos elementos de jogos implementados), por exemplo, avaliar o projeto de gamificação para verificar se o mesmo foi desenvolvido seguindo algum modelo (e.g., métodos, abordagem, frameworks, ferramenta, entre outros) antes ou durante a fase de implementação.

Diferentemente dos métodos tradicionais de avaliação, o uso de gamificação permite a avaliação por meio de métricas que estão associadas aos elementos de jogos, e, portanto, podem ser observadas e coletadas diretamente dos elementos de jogos implementados em um ambiente gamificado. Por exemplo, para avaliar o desempenho de um aluno, os pontos ou a posição geral em uma tabela de classificação pode ser utilizado como um indicador/métrica para inferir sobre o desempenho geral do aluno em uma determinada atividade ou unidade curricular. A taxonomia proposta por Toda et al., (2019) apresenta os elementos de jogos mais utilizados no âmbito educacional distribuído em cinco categorias. Desse modo, para mensurar o desempenho de um aluno, os elementos da categoria performance são alguns dos elementos que podem ser usados para inferir sobre o desempenho dos alunos. A seguir serão descritos alguns exemplos de como utilizá-los como métricas em uma avaliação.

- Emblemas, medalhas, troféus e conquistas: esses elementos são responsáveis por gerar um feedback extrínseco sempre que o aluno completa uma ação ou um conjunto de ações. Nesse contexto, o avaliador pode analisar a quantidade de emblemas que o aluno obteve para verificar se o mesmo completou as atividades propostas assim como analisar se houve interação com o ambiente. Por exemplo, se o ambiente gamificado recompensar com um emblema chamado "The Finisher" sempre que um aluno finalizar todas as atividades, o avaliador pode utilizar esse emblema para constatar se o aluno completou todas as tarefas.

- Pontuação, níveis e progresso: esses elementos estão entre os mais comuns implementados em ambientes gamificados. A pontuação, dependendo de sua implementação, permite que o avaliador analise o desempenho do aluno no ambiente gamificado; da mesma forma que os níveis (e.g., nível do personagem ou nível de habilidade) e o progresso (e.g., nível de fase, etapa) podem ser utilizados como métricas para inferir sobre o progresso ou desempenho geral.

Os elementos discutidos acima são apenas alguns exemplos de elementos que podem ser utilizados para uma análise quantitativa/qualitativa, entretanto, a avaliação dos elementos não precisa se limitar apenas a avaliação de elementos da categoria performance. Elementos da categoria Social, por exemplo, podem ser analisados para avaliar a interação dos usuários com a plataforma ou entre usuários. Nesse sentido, para utilizar os elementos de jogos como indicadores de uma avaliação, é imprescindível que o avaliador tenha conhecimento do seu ambiente gamificado e saiba como este foi 
projetado, possibilitando que o mesmo realize uma avaliação mais rica e dinâmica, utilizando os elementos do ambiente como métricas de medição para sua avaliação.

\subsection{Dimensão 2 - Quando Avaliar?}

- Diretriz 2: Analisar o contexto de aplicação da gamificação e planejar a avaliação com base neste contexto.

O objetivo dessa dimensão é fornecer ao avaliador algumas orientações relacionadas ao contexto em que a gamificação foi aplicada para auxiliá-lo no planejamento da avaliação. Para auxiliar na compreensão do contexto e no planejamento da avaliação, essa dimensão abordará quatros elementos encontrados na literatura de gamificação que podem ser úteis para a construção da avaliação.

\subsubsection{Público-Alvo}

Identificar o público-alvo/participantes e as características dos usuários é um fator fundamental no processo avaliativo, uma vez que as características dos usuários podem influenciar tanto no planejamento quanto no resultado da avaliação. Características como a idade, sexo, personalidade, cultura e preferências em jogos podem impactar na experiência proporcionada ao usuário (Klock et al., 2016). Por exemplo, a idade é um fator que poderá, de alguma forma, limitar o uso de determinadas técnicas de avaliação com base em questionários, dado que a idade do público-alvo é um ponto fundamental para que algumas questões possam ser compreendidas. Nesse sentido, há de se considerar possíveis adaptações, ou ainda, outras técnicas de avaliação.

Os métodos utilizados para identificar o perfil do jogador têm sido herdados ou inspirados na literatura da área de design de jogos (Andrade, 2018). Esses métodos vem sendo uma tendência na área de gamificação adaptativa/inteligente (i.e., ambientes gamificados que se adaptam ou são adaptados para o usuário baseado em suas características). Entre as abordagens para identificar o perfil de jogador, pode-se destacar o HEXAD (Tondello et al., 2019), que descreve perfis de jogadores especificamente para ambientes gamificados. Na avaliação, os métodos de classificação podem ser utilizados para inferir sobre um determinado resultado.

\subsubsection{Ambiente Gamificado}

Este elemento refere-se ao tipo de ambiente em que os elementos de gamificação foram implementados. No âmbito educacional podem-se destacar três diferentes tipos de configurações para elaborar uma experiência com uso de gamificação: presenciais, virtuais e híbridos. Os ambientes gamificados presenciais são aqueles que acontecem no âmbito "real" através de estratégias lúdicas com a aplicação de elementos de jogos em sala de aula. Por exemplo, usar cartolinas para simular uma tabela de lideranças para classificar os alunos que obtiveram êxito em alguma tarefa, atividade ou prova, distribuição de prêmios (e.g., balas, pirulitos, doces) ou medalhas/emblemas quando uma conquista é alcançada pelo aluno (e.g., premiar com uma medalha os alunos que obtiveram nota máxima na disciplina).

Já os ambientes gamificados virtuais abrangem os ambientes que implementam algum tipo de elemento de jogo por meio de alguma aplicação. No âmbito educacional, normalmente o uso de gamificação ocorre através de ambientes virtuais de aprendizagem, moocs e também aplicações mobile. Já existem serviços gratuitos que oferecem plataformas gamificadas, por exemplo, o Moodle fornece acesso gratuito à diversos plugins $^{1}$ que podem ser configurados para implementar elementos de jogos na plataforma.

1 Página de Plugins relacionados com gamificação para a plataforma Moodle: https://moodle.org/plugins/browse.php?list=set\&id=88 
Enquanto que os ambientes gamificados híbridos, como o próprio nome sugere, referemse aos ambientes que promovem a gamificação tanto no âmbito presencial quanto no âmbito virtual. o Classcraft ${ }^{2}$ é uma plataforma que simula um ambiente RPG educacional online e gratuito e permite que o professor configure o ambiente de acordo com suas necessidades, independente da disciplina ou conteúdo. A aplicação possui diversas abordagens que promovem a interação do aluno entre o ambiente virtual e o ambiente real (e.g., recompensar o aluno com 15 minutos de intervalo da aula quando alcançar uma determinada conquista).

\subsubsection{Equipamentos e materiais}

Deve-se verificar que recursos são necessários e estão disponíveis para realizar a avaliação bem como a coleta dos dados. Por exemplo, para coletar logs em um ambiente virtual de aprendizagem pode ser necessário a implementação de um dashboard ou o conhecimento especializado de um especialista para consultar os dados em uma base dados. Da mesma forma, em um ambiente presencial, caso sejam feitos registros através de câmeras é preciso identificar quantas câmeras serão necessárias e onde serão instaladas, uma vez que dependendo do posicionamento desses aparelhos alguns usuários podem se sentir desconfortáveis, necessitando um maior planejamento no ambiente para evitar ou minimizar possíveis desconfortos (Rogers et al., 2013).

\subsubsection{Cronograma e Orçamento}

O último elemento de contexto corresponde as limitações relacionadas ao cronograma e ao orçamento. Em primeiro lugar, deve-se ter em mente a quantidade de tempo necessária para realização da avaliação, por exemplo, a avaliação acontecerá em um encontro, durante um determinado período de tempo ou ainda durante todo o período letivo? As avaliações em um encontro, comumente, são menos complexas e exigem menor esforço de planejamento por parte do avaliador, possibilitando um diagnóstico rápido da experiência. Por outro lado, as avaliações longas possibilitam uma análise mais precisa e detalhada sobre a experiência, já que permite investigar os efeitos da gamificação a longo prazo sobre os alunos.

Já as questões orçamentárias referem-se ao custo que pode haver para a realização da avaliação, restrições orçamentárias podem impedir a compra de materiais para registro (i.e., materiais para gravação ou anotações) pagamento de servidores, entre outros. Dependendo do contexto, se a gamificação for desenvolvida para dispositivos móveis, é preciso verificar quantos usuários possuem dispositivos e a compatibilidade com estes aparelhos. Ainda, é preciso considerar usuários que não possuem dispositivos para usufruir da gamificação, nestes casos, o planejamento da atividade com gamificação e, consequentemente, da avaliação, deve ser pensada para que todos os usuários possam usufruir da atividade e participar da mesma forma.

\subsubsection{Quando avaliar?}

Baseado nos elementos discutidos acima, a avaliação pode ocorrer em três momentos distintos. O primeiro momento de avaliação ocorre antecedendo a aplicação da gamificação (no início do ciclo do design, ainda durante o desenvolvimento do ambiente gamificado), podendo auxiliar para verificar se o desenvolvimento do ambiente está atendendo a um padrão de qualidade ou modelo referência. $\mathrm{O}$ segundo momento de avaliação pode ocorrer durante a aplicação da gamificação, isto é, concomitantemente ao uso da gamificação, possibilitando o feedback e análise dos dados em tempo real, permitindo que o avaliador-verifique os efeitos da gamificação e faça adaptações no

${ }_{2}$ Acesso para a página Inicial do Google ClassCraft: https://www.classcraft.com/pt/. 
ambiente ou na estratégia, caso julgue necessário. Por fim, a avaliação pode ocorrer após a prática com a gamificação, sendo usualmente utilizada para solicitar a opinião dos usuários em relação a sua experiência, assim como verificar se os objetivos ou metas foram alcançados.

\subsection{Como avaliar?}

- Diretriz 3. Definir uma ou mais técnicas de avaliação, e, quando possível, apoiar-se em técnicas já validadas

As técnicas de avaliação determinam os procedimentos utilizados para a obtenção dos dados ao longo da avaliação. Neste framework, serão abordadas três categorias de técnicas de avaliação, sendo estas: Técnicas objetivas/empíricas, Técnicas preditivas e Técnicas prospectivas (ONGARO e CANAL, 2004). É importante destacar que os termos utilizados para essa classificação foram desenvolvidos para o contexto de avaliação em IHC, no entanto, os exemplos que serão apresentados em cada categoria foram baseados ou extraídos, exclusivamente, em estudos no campo de gamificação.

As Técnicas Objetivas/Empíricas baseiam-se em dados provenientes da interação do usuário com o ambiente gamificado; tipicamente, essas técnicas são aplicadas em ambientes naturais (e.g., na sala de aula ou ambientes virtuais) e abrangem o uso de atividades gamificadas ( $e . g$, aplicação de testes/provas, tarefas, atividades para os alunos em sala de aula utilizando estratégias gamificadas), observação e monitoramento. Já as Técnicas Prospectivas são aquelas baseadas na aplicação de questionários e entrevistas com os usuários e, comumente, são empregadas para avaliar a satisfação do usuário em relação ao ambiente gamificado.

Por fim, as Técnicas Preditivas são aquelas que se baseiam em algum tipo de previsão, podendo ser baseada em teorias, heurísticas, modelos, instrumentos, entre outros. Nessa abordagem de avaliação não é necessário envolver os usuários finais. $\mathrm{Na}$ literatura já existem alguns frameworks que podem ser utilizados para uma avaliação preditiva, como o Octalysis de Chou (2019) que permite a avaliação do design com base em oito campos de motivação. A avaliação preditiva também pode ser feita baseada em taxonomias, por exemplo, a taxonomia de Toda et al., (2019) pode ser empregada para analisar o design do ambiente com base nos elementos que foram implementados. Por fim, inspirado em métodos de IHC, a avaliação também pode ser feita com base em heurísticas, como exemplo, Roy e Zanam (2017) elaboraram um conjunto de 9 heurísticas que podem ser utilizadas para avaliar um ambiente gamificado em formato de checklist.

Normalmente, as técnicas preditivas exigem maior conhecimento do avaliador, no entanto, para aumentar a confiabilidade dos resultados, essas técnicas podem ser realizadas por mais de um avaliador. Em decorrência da limitação de páginas, os exemplos para cada uma das técnicas discutidas foram alocados como material suplementar e podem ser acessados no seguinte link: https://bityli.com/Va25r1.

\subsection{Como Lidar com Questões Éticas?}

- Diretriz 4: Considerar questões éticas quando envolver outros usuários na avaliação.

A Association for Computer Machinery (ACM) e outras organizações fornecem códigos éticos especialmente quando a atividade envolver outros seres humanos (Rogers et al., 2013). Entre as questões éticas mais comuns está o direito à privacidade, isto é, o nome dos participantes da avaliação não deve ser associado aos dados coletados ou compartilhados em relatórios, a menos que haja permissão do participante. Entre as recomendações indicadas por Rogers et al., (2013) estão: (1) Informar aos participantes os objetivos do estudo e exatamente o que eles devem esperar, caso aceitem participar do 
experimento; (2) Certificar-se de explicar as informações demográficas, financeiras, de saúde, ou outras informações delicadas que os usuários forneceram ou que ficaram evidentes durante o estudo; (3) Informar aos usuários para que saibam que estão livres para deixar a avaliação no momento em que não estiverem mais se sentindo confortáveis com o procedimento; (4) Evitar incluir citações ou descrições que revelem inadvertidamente a identidade dos participantes; (5) Pedir permissão aos participantes com antecedência para citá-los, destacando que suas identidades não serão reveladas.

\subsection{Como Relatar? \\ - Diretriz 5. Coletar, analisar e apresentar os resultados de forma clara e concisa.}

Esta dimensão tem como objetivo auxiliar o avaliador a interpretar os dados coletados considerando alguns fatores intrínsecos de experiências que promovem o uso gamificação em contextos educacionais. Para tal, a dimensão foi separada em três segmentos: o primeiro aborda o processo de coleta de dados, já o segundo expõe algumas variáveis e fatores que podem impactar na análise e interpretação dos dados coletados e, por último, são apresentados alguns métodos para a apresentação dos dados. Os três segmentos também podem ser consultados no material suplementar através do link: https://bit.ly/3GEFU89.

\section{Discussão e Conclusão}

Nos últimos anos a gamificação tornou-se um tópico popular e amplamente discutido pelos pesquisadores, permeando principalmente o campo da educação. No entanto, foi possível observar que a avaliação da gamificação na educação, apesar de sua importância, ainda é uma área pouco explorada na literatura especializada. Ainda que existam frameworks que exploram aspectos de avaliação como parte de sua estrutura como pode ser visto nos trabalhos de Nah et al., (2013), Mora et al., (2015) e Klock et al., (2016), é razoável destacar que estes foram desenvolvidos a partir de um escopo específico. Como diferencial, O GAMIEFECTS foi desenvolvido em sua totalidade centrado no processo de avaliação e pode ser utilizado por avaliadores sem conhecimento especializado em gamificação. O GAMIEFECTS tem como objetivo principal auxiliar e orientar o avaliador ao longo de um processo de avaliação da gamificação em contextos educacionais e é recomendado para realização de estudos empíricos com ou sem abordagens experimentais, estudos de caso ou como uma ferramenta para guiar a construção de avaliações instrucionais em sala de aula ou de design de gamificação. Como trabalho futuro, atendendo a etapa de avaliação do DSRM, pretende-se planejar e desenvolver uma validação do framework por meio de estudos de caso, assim como realizar uma investigação com especialistas na área de gamificação buscando investigar atributos específicos pertinentes de um framework, como sua utilidade, usabilidade, compreensibilidade, entre outros critérios, e a partir deste diagnóstico, se necessário, realizar melhorias em sua estrutura.

\section{Referências}

Andrade, F. R. H. (2018). Gamificação personalizada baseada no perfil do jogador (Doctoral dissertation, Universidade de São Paulo).

Chou, Y. K. (2019). Actionable gamification: Beyond points, badges, and leaderboards. Packt Publishing Ltd.

Costa, R. M., \& SILVA, J. (2014). Design Research é uma metodologia de aplicação prática. In: III Congresso Internacional de Pós-Graduações e Pesquisas Latinos em Administração e Estudos Organizacionais A administração e o Estudos Organizacionais no contexto Latino-Americano. 
Dal Ongaro, E., \& Canal, A. P. (2004). Técnicas de usabilidade aplicadas aos softwares da empresa zipline tecnologia ltda. Disciplinarum Sciential Naturais $\boldsymbol{e}$ Tecnológicas, 5(1), 163-183.

DE-MARCOS, Luis et al. An empirical study comparing gamification and social networking on e-learning. Computers \& education, v. 75, p. 82-91, 2014.

Dichev, C., \& Dicheva, D. (2017). Gamifying education: what is known, what is believed and what remains uncertain: a critical review. International journal of educational technology in higher education, 14(1), 1-36.

HANUS, Michael D.; FOX, Jesse. Assessing the effects of gamification in the classroom: A longitudinal study on intrinsic motivation, social comparison, satisfaction, effort, and academic performance. Computers \& education, v. 80, p. 152-161, 2015.

Klock, A. C. T., Gasparini, I., \& Pimenta, M. S. (2016). 5W2H Framework: a guide to design, develop and evaluate the user-centered gamification. In: Proceedings of the 15th Brazilian Symposium on Human Factors in Computing Systems (pp. 1-10).

KLOCK, Ana Carolina Tomé et al. Does gamification matter? A systematic mapping about the evaluation of gamification in educational environments. In: Proceedings of the 33rd Annual ACM Symposium on Applied Computing. 2018. p. 2006-2012.

Mora, A., Zaharias, P., González, C., \& Arnedo-Moreno, J. (2015). Fraggle: a Framework for agile gamification of learning experiences. In: International Conference on Games and Learning Alliance (pp. 530-539). Springer, Cham.

Nah, F. F. H., Telaprolu, V. R., Rallapalli, S., \& Venkata, P. R. (2013). Gamification of education using computer games. In: International Conference on Human Interface and the Management of Information (pp. 99-107).

Ogawa, A., Klock, A. C. T., \& Gasparini, I. (2016). Avaliação da gamificação na área educacional: um mapeamento sistemático. In: Brazilian Symposium on Computers in Education (Simpósio Brasileiro de Informática na Educação-SBIE) (Vol. 27, No. 1, p. 440).

Peffers, K., Tuunanen, T., Rothenberger, M. A., \& Chatterjee, S. (2007). A design science research methodology for information systems research. Journal of management information systems, 24(3), 45-77.

Reinheimer, W. S., \& Medina, R. D. (2020). Gamification in the Educational Context: A Systematic Mapping of Literature with a Focus on the Evaluation of Gamification. RENOTE, 18(2), 398-407.

Rogers, Y., Sharp, H., \& Preece, J. (2013). Design de interação. Bookman Editora. Toda, A. M., Oliveira, W., Klock, A. C., Palomino, P. T., Pimenta, M., Gasparini, I., ... \& Cristea, A. I. (2019, July). A taxonomy of game elements for gamification in educational contexts: Proposal and evaluation. In 2019 IEEE 19th International Conference on Advanced Learning Technologies (ICALT) (Vol. 2161, pp. 84-88). IEEE.

TODA, Armando M. et al. Frameworks para o planejamento da gamificaçao em contextos educacionais-uma revisao da literatura nacional. RENOTE-Revista Novas Tecnologias na Educação, v. 16, n. 2, p. 505-514, 2018.

Tondello, G. F., Mora, A., Marczewski, A., \& Nacke, L. E. (2019). Empirical validation of the gamification user types hexad scale in English and Spanish. International Journal of Human-Computer Studies, 127, 95-111.

Van Roy, R., \& Zaman, B. (2017). Why gamification fails in education and how to make it successful: Introducing nine gamification heuristics based on self-determination theory. In Serious Games and edutainment applications (pp. 485-509). Springer, Cham. 